\title{
DIFFERENTIABLE TCHEBYCHEFF SUBSPACES AND HERMITE INTERPOLATION
}

\author{
WERNER HAUSSMANN
}

\section{Introduction}

Let $m, n \in N, 1 \leqq m \leqq n$, and let $E_{n}=\left(\varepsilon_{\mu \nu}\right)_{1 \leqq \mu \leqq m, 0 \leqq v \leqq n-1}$ be an incidence matrix (cf. Schoenberg [12], Mäkelä-Nevanlinna-Sipilä [8]), i.e. $\varepsilon_{\mu \nu}=0$ or 1, and $\sum_{\mu, \nu} \varepsilon_{\mu \nu}=n$. Suppose, for simplicity, that no row is composed only of zeros. Given an interval $I \subset \boldsymbol{R}$ with nonvoid interior $\stackrel{\circ}{I}$, interpolation nodes $t_{1}<t_{2}<\ldots<t_{m}$ in $I$, and an $n$-dimensional subspace $U \subset C^{r}(I), r \in N_{0}:=N \cup\{0\}$, then the incidence matrix $E_{n}$ gives rise to the following interpolation problem: Does there exist a $u \in U$ satisfying

$$
u^{(v)}\left(t_{\mu}\right)=a_{\mu v}
$$

for all $(\mu, v)$ such that $\varepsilon_{\mu \nu}=1$ with arbitrarily given data $a_{\mu \nu} \in \boldsymbol{R}$ ? The problem of unique solvability of this Birkhoff type interpolation problem has been treated by several authors for the case $U=\Pi_{n-1}$ (polynomials of degree not exceeding $n-1$ ), see e.g. the surveys of Sharma [13] or Lorentz [7].

In a recent paper, Mäkelä-Nevanlinna-Sipilä [8] put the following question: Given a fixed type of incidence matrix $E_{n}$, what are the properties of $U \subset C^{r}(I)$ necessary and sufficient that the interpolation problem (1) be uniquely solvable (with respect to certain special or arbitrary nodes)? They considered various types of spaces $U$ generalizing results of Matthews [9], Ikebe [3] (the latter treated general Birkhoff interpolation problems) as well as of [1]. In most of their results MäkeläNevanlinna-Sipilä [8] make use of "polynomial like" spaces $U$ for which the dimension of $U$ is reduced by differentiation.

It is the topic of the present paper to investigate those $n$-dimensional subspaces $U \subset C^{r}(I), r \in N$, whose dimension is not necessarily reduced by differentiation in order to get results for Hermite interpolation incidence matrices. In [1] we showed that the osculatory Hermite interpolation problem is uniquely solvable with respect to differentiable Tchebycheff subspaces. When proving this, one of the main problems was that - because of the lack of sufficient differentiability - the usual multiplicity notion for zeros of a function does not work. Thus we have to use a certain multi- 
plicity notion for $r$-times differentiable functions in order to treat the general Hermite interpolation in $C^{r}(I)$ here (cf. Mäkelä-Nevanlinna-Sipilä [8]). Then we give a characterization of Hermite subspaces which enables us to prove the main theorem on Hermite interpolation in Section 3. Some of these results were announced in [2]. In addition we can characterize weakly differentiable Tchebycheff subspaces $U \subset C^{r}(I)$ of dimension $n$ by means of a certain class of incidence matrices.

Other investigations on Hermite interpolation by nonpolynomials are due to Polya [10] and Karlin-Studden [6]. Some results on Birkhoff interpolation with respect to nonpolynomials go back to Karlin-Karon [4, 5].

\section{A characterization of Hermite subspaces}

In order to prove the results in Section 3 we need the notion of a Hermite subspace as well as a characterization of Hermite spaces which is established in this section.

Let $n \in N, r \in N_{0}$, and $I \subset R$ an interval $(i \neq \emptyset)$. An $n$-dimensional subspace $U \subset C^{r}(I)$ is called a Hermite subspace of $C^{r}(I)$ provided that for any $m \in N, m \leqq n$, any $\left(\alpha_{1}, \ldots, \alpha_{m}\right) \in N_{0}^{m}$ satisfying $\max _{1 \leqq \mu \leqq m} \alpha_{\mu} \leqq r$ and $\sum_{1 \leqq \mu \leqq m}\left(\alpha_{\mu}+1\right)=n$, and arbitrary interpolation nodes $t_{1}<t_{2}<\ldots<t_{m}$ in $I$ the following Hermite interpolation problem is uniquely solvable:

Given any $a_{\mu \tau} \in \boldsymbol{R}\left(0 \leqq \tau \leqq \alpha_{\mu}, 1 \leqq \mu \leqq m\right)$, does there exist a $u \in U$ satisfying

$$
u^{(\tau)}\left(t_{\mu}\right)=a_{\mu \tau} \quad\left(0 \leqq \tau \leqq \alpha_{\mu}, 1 \leqq \mu \leqq m\right) ?
$$

The set of all $n$-dimensional Hermite subspaces of $C^{r}(I)$ will be denoted by $\mathscr{H}_{n}^{r}(I)$.

In order to characterize Hermite subspaces we need the following notion of multiplicity for zeros of functions in $C^{r}(I)$ which will be defined inductively (see Mäkelä-Nevanlinna-Sipilä [8] and [2]):

(i) Let $x \in C^{r}(I), t \in I$, then define

$$
z_{0}(x, t):=\left\{\begin{array}{lll}
1 & \text { if } & x(t)=0 \\
0 & \text { if } & x(t) \neq 0
\end{array}\right.
$$

and, for $1 \leqq \varrho \leqq r$ :

$$
z_{\varrho}(x, t):=\left\{\begin{array}{lll}
z_{\varrho-1}\left(x^{\prime}, t\right)+1 & \text { if } & x(t)=0 \\
0 & \text { if } & x(t) \neq 0
\end{array}\right.
$$

Then $\mathscr{Z}_{r}(x):=\sum_{t \in I} z_{r}(x, t)$ is the number of all zeros of $x \in C^{r}(I)$ where multiple zeros are counted according to this (weak) multiplicity notion. 
(ii) In order to count multiplicities of zeros in a strict sense, let again $x \in C^{r}(I)$, $t \in I$. Then define (cf. Rice [11])

$$
\tilde{z}_{0}(x, t):= \begin{cases}2 & \begin{array}{l}
\text { if } x(t)=0, t \in \stackrel{\circ}{1}, \\
\text { not change sign } t \text { is an isolated zero where } x \text { does }
\end{array} \\
1 & \text { if } x(t)=0, \text { and } \tilde{z}_{0}(x, t) \neq 2 \\
0 & \text { if } x(t) \neq 0\end{cases}
$$

and, for $1 \leqq \varrho \leqq r:$

$$
\tilde{z}_{\varrho}(x, t):=\left\{\begin{array}{lll}
\tilde{z}_{\varrho-1}\left(x^{\prime}, t\right)+1 & \text { if } & x(t)=0 \\
0 & \text { if } & x(t) \neq 0 .
\end{array}\right.
$$

The number of all zeros counted with this strict multiplicity notion will be denoted by $\tilde{\mathscr{Z}}_{r}(x):=\sum_{t \in I} \tilde{z}_{r}(x, t)$.

Note that we have $0 \leqq z_{r}(x, t) \leqq r+1,0 \leqq \tilde{z}_{r}(x, t) \leqq r+2$ for $x \in C^{r}(I)$ and $t \in I$. In addition, for $\tilde{z}_{r}(x, t) \leqq r+1$, we have $\tilde{z}_{r}(x, t)=z_{r}(x, t)$.

With these preparations we are able to prove the following theorem without the use of determinants by interpolation theoretical means only:

Theorem 1. Let $U \subset C^{r}(I)$ be an n-dimensional subspace $\left(r \in N_{0}, n \in N, I \subset R\right.$ an interval with nonvoid interior). Then these assertions are equivalent:

(i) $U$ is an $n$-dimensional Hermite subspace of $C^{r}(I)$,

(ii) For any $u \in U, u \neq 0$, we have: $\mathscr{Z}_{r}(u) \leqq n-1$,

(iii) For any $u \in U, u \neq 0$, we have: $\tilde{\mathscr{Z}}_{r}(u) \leqq n-1$.

Proof. (i) $\Rightarrow$ (ii): Let $U$ be an $n$-dimensional subspace of $C^{r}(I)$, and suppose there exists a $u_{0} \in U, u_{0} \neq 0$, such that $\mathscr{Z}_{r}\left(u_{0}\right) \geqq n$. We can assume that $u_{0}$ possesses only a finite number of zeros $t_{1}, \ldots, t_{k} \in I(k \leqq n-1)$; otherwise $u_{0}$ would be a nontrivial solution of the Lagrange interpolation problem $u\left(s_{v}\right)=0$ for $n$ or more points $s_{v} \in I$.

Thus let $z_{r}\left(u_{0}, t_{\varkappa}\right)=\beta_{\varkappa}+1(1 \leqq \varkappa \leqq k)$, and define

$$
m:=\min \left\{l \in N: \sum_{1 \leqq \lambda \leqq l} z_{r}\left(u_{0}, t_{\lambda}\right) \geqq n\right\} .
$$

Now put $\alpha_{\mu}=\beta_{\mu}(1 \leqq \mu \leqq m-1)$, and $\alpha_{m}=n-\sum_{1 \leqq \mu \leqq m-1} z_{r}\left(u_{0}, t_{\mu}\right)-1$. Then the Hermite interpolation problem

$$
u^{(\tau)}\left(t_{\mu}\right)=0 \quad\left(0 \leqq \tau \leqq \alpha_{\mu}, 1 \leqq \mu \leqq m\right)
$$

has the nontrivial solution $u_{0}$ which contradicts the fact that a Hermite subspace yields a unique solution of any Hermite interpolation problem involving derivative conditions up to order $r$.

(ii) $\Rightarrow$ (i): Now suppose $\mathscr{Z}_{r}(u) \leqq n-1$ for any $u \in U, u \neq 0$, and let an interpolation problem of type (2) with nodes $t_{1}<t_{2}<\ldots<t_{m}$ in $I$ be given. It suffices to show that the homogeneous problem corresponding to (2) has the trivial solution only. 
Any solution $u \in U$ of this problem possesses zeros of weak multiplicities $\geqq \alpha_{\mu}+1$ at $t_{\mu}$, thus we have

$$
\sum_{t \in I} z_{r}(u, t) \geqq \sum_{1 \leqq \mu \leqq m} z_{r}\left(u, t_{\mu}\right) \geqq \sum_{1 \leqq \mu \leqq m}\left(\alpha_{\mu}+1\right)=n,
$$

hence $u=0$ by (ii). This yields (i).

Since (iii) $\Rightarrow$ (ii) is obvious, we only have to prove the converse direction. It turns out to be convenient to show that the equivalent statements (i) and (ii) together imply (iii).

(i) + (ii) $\Rightarrow$ (iii): Assume there is a $u_{0} \in U, u_{0} \neq 0$, such that $\tilde{\mathscr{Z}}_{r}\left(u_{0}\right) \geqq n$. Since (ii) holds, $u_{0}$ has at most $k \leqq n-1$ zeros $t_{1}<t_{2}<\ldots<t_{k}$ in $I$. The assertion (ii) implies, too, that there is at least one of the $t_{\mu}$ 's, say $t_{\mu_{0}}$, such that $\tilde{z}_{r}\left(u_{0}, t_{\mu_{0}}\right)=r+2$, which means that

Define

$$
M^{\prime}:=\left\{\varkappa: 1 \leqq \varkappa \leqq k, \tilde{z}_{r}\left(u_{0}, t_{\varkappa}\right)=r+2\right\} \neq \emptyset .
$$

$$
\sigma_{\varkappa}:=\operatorname{sign}_{t \in U\left(t_{\varkappa}\right)} u_{0}^{(r)}(t) \quad \text { for } \quad x \in M^{\prime}
$$

where $U\left(t_{\chi}\right):=\left\{t \in I: t_{\chi}-\varepsilon<t<t_{\chi}+\varepsilon\right\} \backslash\left\{t_{\chi}\right\}$ with a sufficiently small $\varepsilon>0$ so that $\sigma_{\varkappa}$ is well defined (i.e. the restriction of $u_{0}^{(r)}$ to $U\left(t_{\varkappa}\right)$ has constant sign). Let $M:=\{1, \ldots, k\} \backslash M^{\prime}$, and define $v_{0} \in U$ as the unique solution of the following Hermite interpolation problem (according to (i)):

$$
\begin{aligned}
& v_{0}^{(\alpha)}\left(t_{\varkappa}\right)=0 \quad \text { for } \quad x \in M, \quad 0 \leqq \alpha \leqq z_{r}\left(u_{0}, t_{\varkappa}\right)-1 \text {, } \\
& v_{0}^{(\alpha)}\left(t_{x}\right)=0 \quad \text { for } \quad x \in M^{\prime}, \quad 0 \leqq \alpha \leqq r-1 \text {, } \\
& v_{0}^{(r)}\left(t_{\chi}\right)=-\sigma_{\chi} \text { for } \quad \varkappa \in M^{\prime} \text {, }
\end{aligned}
$$

and, since $q:=\sum_{x \in M} z_{r}\left(u_{0}, t_{\varkappa}\right)+\sum_{\varkappa \in M^{\prime}}(r+1)<n$, choose $p:=n-q$ points $s_{\pi}$ different from each other and from the $t_{\varkappa}$ 's, and complete the interpolation conditions above by

$$
v_{0}\left(s_{\pi}\right)=0 \text { for } 1 \leqq \pi \leqq p .
$$

Since $M^{\prime} \neq \emptyset$, we have $v_{0} \neq 0$. Now we consider $w_{0}:=u_{0}+\eta v_{0}$ with $\eta>0$ sufficiently small, thus $w_{0} \neq 0$. By construction, we have

and

$$
z_{r}\left(w_{0}, t_{\varkappa}\right) \geqq z_{r}\left(u_{0}, t_{\varkappa}\right) \text { for } \quad x \in M
$$

$$
z_{r}\left(w_{0}, t_{x}\right) \geqq r \quad \text { for } \quad x \in M^{\prime},
$$

and with a sufficiently small $\eta>0$ for any $x \in M^{\prime}$ there are points $\tau_{x}^{+}$and $\tau_{x}^{-}$(satisfying $\tau_{\varkappa}^{-}<t_{\chi}<\tau_{\varkappa}^{+}$) in a neighbourhood of $t_{\varkappa}$ such that $\left\{t: \tau_{\varkappa}^{-}<t<\tau_{\varkappa}^{+}\right\} \supsetneqq t_{\mu}, \tau_{\mu}^{+}, \tau_{\mu}^{-}, s_{\pi}$ for $1 \leqq \mu \leqq k, \mu \neq \varkappa$, and $1 \leqq \pi \leqq p$, satisfying

Hence we get

$$
z_{r}\left(w_{0}, \tau_{\varkappa}^{+}\right) \geqq 1, \quad z_{r}\left(w_{0}, \tau_{\varkappa}^{-}\right) \geqq 1 .
$$

$$
\mathscr{Z}_{r}\left(w_{0}\right) \geqq \sum_{x \in M} z_{r}\left(w_{0}, t_{x}\right)+\sum_{x \in M^{\prime}}(r+2)=\tilde{\mathscr{Z}}_{\boldsymbol{r}}\left(u_{0}\right) \geqq n
$$


while $w_{0} \neq 0$. Since $w_{0} \in U$, this contradicts (ii). Thus $\tilde{\mathscr{Z}}_{r}(u) \leqq n-1$ for all $u \in U$, $u \neq 0$.

We remark that Theorem 1 also can be obtained with the aid of results due to Mäkelä-Nevanlinna-Sipilä [8] using a characterization of Hermite subspaces in terms of determinant conditions.

If we assume $U$ being an $n$-dimensional space of sufficiently differentiable functions (and not only $U \subset C^{r}(I)$ ) then Theorem 1 reduces to a result of KarlinStudden [6].

\section{Differentiable Tchebycheff subspaces}

We are going to prove sufficient criteria to guarantee unique Hermite interpolation within the framework of differentiable Tchebycheff subspaces. The latter ones will be defined by properties of the number of zeros of a function without using multiplicities of zeros. But when proving the existence and uniqueness Theorem 3 the multiplicities introduced in Section 2 will play an important role.

Given a subspace $U \subset C^{r}(I), I \subset \boldsymbol{R}$ an interval satisfying $i \neq \emptyset$, we define $U^{(\varrho)}:=\{w: w$ is derivative of order $\varrho$ of some $u \in U\}$ for $1 \leqq \varrho \leqq r \in N$. In the case $\varrho=0$ we have $U^{(0)}:=U$. Finally, let $n_{\varrho}:=\operatorname{dim} U^{(\varrho)}$ for $1 \leqq \varrho \leqq r$, and $n_{0}:=n=$ $\operatorname{dim} U$.

Definition 2. Let $n, r \in N, I \subset \boldsymbol{R}$ an interval with nonvoid interior, and $U \subset C^{r}(I)$ an $n$-dimensional subspace.

(i) $U$ is called an r-times differentiable Tchebycheff subspace of dimension $n$ if for $0 \leqq \varrho \leqq r$ the following condition holds: Any $u \in U^{(\varrho)}, u \neq 0$, possesses at most $n_{\varrho}-1$ zeros in $I$ (without counting multiplicities).

(ii) An $r$-times differentiable Tchebycheff subspace $U$ of dimension $n$ is called

$\alpha$ ) an $r$-times strictly differentiable Tchebycheff subspace if

$$
r \leqq n \quad \text { and } \quad \operatorname{dim} U^{(\varrho)}=n-\varrho \quad(0 \leqq \varrho \leqq r),
$$

$\beta)$ an $r$-times weakly differentiable Tchebycheff subspace if

$$
\operatorname{dim} U^{(\varrho)}=n \quad(0 \leqq \varrho \leqq r) .
$$

In order to describe certain interpolation problems we are going to introduce incidence matrices of type $\mathscr{T}_{n}(s, r)$. Given an incidence matrix $E_{n}=\left(\varepsilon_{\mu \nu}\right)_{1 \leqq \mu \leqq m, 0 \leqq v \leqq n-1}$ (see Section 1), and $s \in N_{0}, r \in N$ such that $0 \leqq s \leqq r \leqq n-1$, then we put

$$
m_{v}:=\left\{\mu: \varepsilon_{\mu v}=1(1 \leqq \mu \leqq m)\right\} \text { for } 0 \leqq v \leqq n-1 .
$$

An incidence matrix $E_{n}$ (or the corresponding interpolation problem with respect to some interpolation nodes $\left.t_{\mu}(1 \leqq \mu \leqq m)\right)$ will be called of type $\mathscr{T}_{n}(s, r)$ if the fol- 
lowing conditions hold:

$$
\begin{aligned}
& m_{0}=m_{1}=\ldots=m_{s-1}=\emptyset, \\
& m_{s} \supset m_{s+1} \supset \ldots \supset m_{r}, \quad m_{s} \neq \emptyset, \\
& m_{r+1}=m_{r+2}=\ldots=m_{n-1}=\emptyset .
\end{aligned}
$$

For $s=0$ we have a Hermite interpolation incidence matrix, whereas $s>0$ yields the incidence matrix of a "shifted" Hermite problem.

We are now coming to state the main result of this paper, where we shall use the following notation. Let $E_{n}$ be an incidence matrix, and $t_{1}<t_{2}<\ldots<t_{m}$ interpolation nodes in the interval $I \subset \boldsymbol{R}$. Then we define

$$
M_{v}:=\left\{t_{\mu}: \varepsilon_{\mu \nu}=1(1 \leqq \mu \leqq m)\right\} \text { for } 0 \leqq v \leqq n-1 .
$$

Thus in the case of an incidence matrix of type $\mathscr{T}_{n}(0, r), 0 \leqq r \leqq n-1$, we have

$$
\emptyset \neq M_{0} \supset M_{1} \supset \ldots \supset M_{r}, \quad M_{r+1}=\ldots=M_{n-1}=\emptyset .
$$

Theorem 3. Let $n \in N, r \in N_{0}$, and $U \subset C^{r}(I)$ be an n-dimensional subspace ( $I \subset \boldsymbol{R}$ an interval satisfying $\stackrel{i}{I \neq \emptyset}$ ). Suppose one of the following conditions holds:

(i) $U$ is an r-times strictly differentiable Tchebycheff subspace of $C^{r}(I)$.

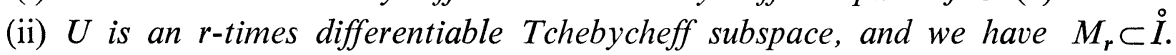

(iii) $U$ is obtained as the restriction to I of an r-times differentiable Tchebycheff subspace $\tilde{U} \subset C^{r}(J)$ of dimension $n$ where $I \subset j$.

Then in either case $U$ is an n-dimensional Hermite subspace of $C^{r}(I)$.

Proof. (i) According to Theorem 1 we have to show (by induction) that if $U$ is an $r$-times strictly differentiable Tchebycheff subspace of dimension $n$ then for any $u \in U, u \neq 0$, we have $\mathscr{Z}_{r}(u) \leqq n-1$.

The case $r=0$ is obvious. Thus let $r>0$, and suppose that the foregoing implication holds true for any $\varrho$-times strictly differentiable Tchebycheff subspace $(0 \leqq \varrho \leqq r-1)$ of any fixed dimension $d \in N$ satisfying $\varrho \leqq d$.

Let $U$ be an $r$-times strictly differentiable Tchebycheff subspace of dimension $n$, and suppose there exists a $u \in U, u \neq 0$, satisfying $\mathscr{Z}_{r}(u)=p \geqq n$. Then $u$ possesses only a finite number of zeros, say $t_{1}, \ldots, t_{l}$, in $I$. Introducing $\beta_{\lambda}:=z_{r}\left(u, t_{j}\right)$ for $1 \leqq \lambda \leqq l$, we have $p=\sum_{1 \leqq \lambda \leqq l} \beta_{\lambda}$. By definition of the weak multiplicity of zeros, the derivative $u^{\prime}$ satisfies

$$
z_{r-1}\left(u^{\prime}, t_{\lambda}\right)=\beta_{\lambda}-1 \quad(1 \leqq \lambda \leqq l) .
$$

In addition, Rolle's theorem yields the existence of $l-1$ further zeros in the open intervals $] t_{\lambda}, t_{\lambda+1}$ [ for $1 \leqq \lambda \leqq l-1$. Thus

$$
\mathscr{Z}_{r-1}\left(u^{\prime}\right) \geqq \sum_{1 \leqq \lambda \leqq l}\left(\beta_{\lambda}-1\right)+l-1=p-1 \geqq n-1 .
$$

Now $U^{(1)}$ is an $(r-1)$-times strictly differentiable Tchebycheff subspace of dimension $n-1$ which yields $u^{\prime}=0$, hence $u=0$, which is a contradiction. Thus $U \in \mathscr{H}_{n}^{r}(I)$. 
The result that condition (i) implies $U$ being an $n$-dimensional Hermite subspace of $C^{r}(I)$ is covered by a theorem of Ikebe [3].

(ii) Again, we prove the assertion by induction with respect to $r$. The case $r=0$ is immediate. Thus assume $r>0$, and suppose the statement holds true for $0 \leqq \varrho \leqq r-1$ and arbitrary dimension of $U$.

Now suppose $U$ is an $r$-times differentiable Tchebycheff subspace of dimension $n$. Let $m$ interpolation nodes $t_{1}<t_{2}<\ldots<t_{m}$ in $I$ be given as well as nonnegative integers $\alpha_{\mu}(1 \leqq \mu \leqq m)$ satisfying $\max _{1 \leqq \mu \leqq m} \alpha_{\mu} \leqq r$ and $\sum_{1 \leqq \mu \leqq m}\left(\alpha_{\mu}+1\right)=n$. It is sufficient to show that the homogeneous interpolation problem

$$
u^{(\tau)}\left(t_{\mu}\right)=0 \quad\left(0 \leqq \tau \leqq \alpha_{\mu}, 1 \leqq \mu \leqq m\right)
$$

has the trivial solution $u_{0}=0$ only.

For any solution $u$ of (3) we have $z_{r}\left(u, t_{\mu}\right) \geqq \alpha_{\mu}+1$. Since $u$ vanishes at $t_{1}, \ldots, t_{m}$ in $I$, by Rolle's theorem, $u^{\prime}$ possesses $m-1$ further zeros $\left.\tau_{\mu} \in\right] t_{\mu}, t_{\mu+1}[(1 \leqq \mu \leqq m-1)$. Thus we have

$$
\tilde{\mathscr{Z}}_{r-1}\left(u^{\prime}\right) \geqq \sum_{1 \leqq \mu \leqq m} \alpha_{\mu}+(m-1)=n-1 .
$$

Now we have to consider two cases:

( $\alpha$ ) If $\operatorname{dim} U^{(1)}=n-1$, then as in the proof of (i) we have $u=0$.

( $\beta$ ) Thus suppose $\operatorname{dim} U^{(1)}=n$. If there is a $t_{\mu_{0}} \in M_{r}(\subset \stackrel{i}{i}$ by hypothesis) such that $\tilde{z}_{r-1}\left(u^{\prime}, t_{\mu_{0}}\right)=r+1$, then we have

$$
\tilde{\mathscr{Z}}_{r-1}\left(u^{\prime}\right) \geqq \sum_{\substack{\mu=1 \\ \mu \neq \mu_{0}}}^{m} \alpha_{\mu}+(m-1)+(r+1) \geqq n
$$

since $\alpha_{\mu_{0}}=r$. By Theorem 1 and the induction hypothesis we have $u^{\prime}=0$, hence $u=0$. Therefore we can assume that for any $t_{\mu} \in M_{r}$ we have

Hence

$$
\tilde{z}_{r-1}\left(u^{\prime}, t_{\mu}\right)=r .
$$

$$
\tilde{z}_{\boldsymbol{r}}\left(u, t_{\mu}\right)=r+1 \text {, }
$$

which (since $M_{r} \subset \stackrel{I}{)}$ implies that

$$
\tilde{z}_{r-1}\left(u, t_{\mu}\right)=r+1 \quad\left(t_{\mu} \in M_{r}\right) .
$$

In addition, for all $1 \leqq \mu \leqq m$

holds true. From this we get

$$
\tilde{z}_{r-1}\left(u, t_{\mu}\right) \geqq \alpha_{\mu}+1
$$

$$
\tilde{\mathscr{Z}}_{r-1}(u)=\sum_{1 \leqq \mu \leqq m} \tilde{z}_{r-1}\left(u, t_{\mu}\right) \geqq \sum_{t_{\mu} \notin M_{r}}\left(\alpha_{\mu}+1\right)+\sum_{t_{\mu} \in M_{r}}(r+1)=\sum_{1 \leqq \mu \leqq m}\left(\alpha_{\mu}+1\right)=n .
$$

Hence $u=0$ by induction hypothesis and Theorem 1 .

(iii) In this case it follows that $M_{r} \subset I \subset \tilde{J}$. Thus (ii) is applicable for $\tilde{U}$ instead of $U$. Hence there exists a unique $\tilde{u} \in \tilde{U}$ which solves the given interpolation problem 
of type $\mathscr{T}_{n}(0, r)$. Its restriction to $I, u:=\tilde{u} \mid I$, is the unique solution of (3) with respect to $U$.

Theorem 3 yields the following generalization of a theorem of Mäkelä-Nevanlinna-Sipilä [8]:

Corollary. Let $U$ be an n-dimensional subspace of $C^{r}(I), I$ an open real interval. Suppose $U, U^{(1)}, \ldots, U^{(r)}$ provide a unique solution for any interpolation problem of type $\mathscr{T}_{n}(0,0)$. Then any interpolation problem of type $\mathscr{T}_{n}(0, r)$ possesses a unique solution with respect to $U$.

\section{A characterization of $r$-times weakly differentiable Tchebycheff subspaces}

The following interpolation theoretical characterization of Tchebycheff subspaces of $C(I)$ is well known (it is an immediate consequence of Theorem 1 for $r=0$ ):

An n-dimensional subspace $U \subset C(I), I$ a nontrivial interval, is a Tchebycheff subspace if and only if any interpolation problem of type $\mathscr{T}_{n}(0,0)$ (i.e. Lagrange interpolation problem) with nodes in I possesses a unique solution.

Now we are going to consider a corresponding characterization of r-times weakly differentiable Tchebycheff subspaces defined on an open interval. The interpolation problems which are needed for this characterization turn out to be of Birkhoff type (see e.g. Schoenberg [12]).

Theorem 4. Let $U \subset C^{r}(I)$ be an n-dimensional subspace, $I \subset \boldsymbol{R}$ an open interval. $U$ is an r-times weakly differentiable Tchebycheff subspace if and only if any interpolation problem of type $\mathscr{T}_{n}(s, r)$ possesses a unique solution with respect to $U$ for any $0 \leqq s \leqq r$.

Proof. Let $U$ be an $r$-times weakly differentiable Tchebycheff subspace of dimension $n$. Then for $0 \leqq s \leqq r$ the spaces $U^{(s)}$ are $(r-s)$-times weakly differentiable Tchebycheff subspaces of dimension $n$. By Theorem 3, any interpolation problem of type $\mathscr{T}_{n}(0, r-s)$ possesses a unique solution with respect to $U^{(s)}$ given any nodes $t_{1}<t_{2}<\ldots<t_{m}$ in $I$ and arbitrary interpolation data. This means that any interpolation problem of type $\mathscr{T}_{n}(s, r)$ has a unique solution with respect to $U$ for $0 \leqq s \leqq r$.

Conversely, let any interpolation problem of type $\mathscr{T}_{n}(s, r)$ be uniquely solvable with respect to $U(0 \leqq s \leqq r)$. Then, in particular, any shifted Lagrange interpolation problem

$$
u^{(s)}\left(t_{v}\right)=a_{v} \quad(1 \leqq v \leqq n)
$$

possesses a unique solution with respect to $U$, hence $U^{(s)}$ is an $n$-dimensional Tchebycheff subspace for $0 \leqq s \leqq r$ which follows from Theorem 1 and Definition 2 .

Examples of $r$-times weakly differentiable Tchebycheff subspaces are given by certain families of exponential functions (see [2]). 


\title{
References
}

[1] Haussmann, W.: On interpolation with derivatives. - SIAM J. Numer. Anal. 8, 1971, 483485.

[2] Haussmann, W.: Hermite-Interpolation mit Čebyšev-Unterräumen. - Numerische Methoden der Approximationstheorie 1, Internat. Schriftenreihe zur Numer. Math. 16, 49-55, Birkhäuser, Basel-Stuttgart, 1972.

[3] IKeBe, Y.: Hermite-Birkhoff interpolation problems in Haar subspaces. - J. Approximation Theory 8, 1973, 142-149.

[4] Karlin, S., and J. M. Karon: Poised and non-poised Hermite-Birkhoff interpolation. Indiana Univ. Math. J. 21, 1972, 1131-1170.

[5] Karlin, S., and J. M. Karon: On Hermite-Birkhoff interpolation. - J. Approximation Theory $6,1972,90-115$.

[6] Karlin, S., and W. J. Studden: Tchebycheff systems: with applications in analysis and statistics. - Interscience Publishers, New York-London-Sydney, 1966.

[7] LoRentz, G. G.: Birkhoff interpolation problem. - University of Texas, Center for Numerical Analysis, Report CNA-103, Austin, 1975.

[8] Mäkelä, M., O. NevanlinNa, and A. H. SipIlä: On some generalized Hermite-Birkhoff interpolation problems. - Ann. Acad. Sci. Fenn. Ser. A I 563, 1974, 1-13.

[9] Matthews, J. W.: Interpolation with derivatives. - SIAM Rev. 12, 1970, 127-128.

[10] Polya, G.: On the mean value theorem corresponding to a given linear homogeneous differential equation. - Trans. Amer. Math. Soc. 24, 1922, 312-324.

[11] Rice, J. R.: The approximation of functions. Vol. 1. Linear theory. - Addison-Wesley, Reading, Mass.-Palo Alto-London, 1964.

[12] Schoenberg, I. J.: On Hermite-Birkhoff interpolation. - J. Math. Anal. Appl. 16, 1966, $538-543$.

[13] Sharma, A.: Some poised and nonpoised problems of interpolation. - SIAM Rev. 14, 1972, $129-151$.

\author{
University of Duisburg \\ Department of Mathematics \\ D-4100 Duisburg 1
}

BRD

Received 12 December 1977 